\title{
Looking at the cancellation rate among renal patients and ways to reduce cancellation at the time of admission and improve utilisation of the theatre list: a quality improvement project
}

\author{
Author: Shayne $\mathrm{Ng}^{\mathrm{A}}$
}

\section{Introduction}

Cancellation on the day of surgery is a major challenge faced by the UK NHS and it was estimated that it costs the NHS $£ 400$ million per year on lost operating theatre time costs. ${ }^{1}$

It was reported that renal patients have a higher than average cancellation rate on the day of surgery (eg elective renal access procedures) due to illness. The standard should be that no patients cancel on day of surgery and all renal patients should be prepared for surgery adequately (eg had dialysis the day before).

\section{Aim}

Our aim was to investigate the cause of the high cancellation rate among renal patients, to reduce the cancellation rate by at least $50 \%$, to improve the patients' experience and to improve utilisation of the theatre list.

\section{Method}

We looked at cancellation rate of renal patients on the day of surgery by looking at elective renal access procedures. Data were collected retrospectively and analysed. We implemented some changes based on findings from two plan, study, do, act (PDSA) cycles. Another PDSA cycle (non-intervention) was carried out 6 months following implementation of the action plans to assess cancellation rate.

\section{Results and discussion}

Prior to the implementation of our action plans, we found $22 \%$ cancellation rate of renal patients on the day of surgery, therefore not meeting the standard. Reasons for cancellation included high blood pressure, unwell patient, hyperkalaemia, uncontrolled bowel movements etc. We implemented some changes, including for patients to be seen by a renal physician 1 day preoperatively to review health status and to maximise condition.
The first PDSA cycle reported a significant improvement in terms of cancellation rate of renal patients. There was a $0 \%$ cancellation rate due to illness, but $6 \%$ cancelled due to administrative error in booking. Further action plans were implemented to reduce the cancellation rate due to administrative error.

Second PDSA cycle (non-intervention) reported further improvement and a $0 \%$ cancellation rate on day of surgery.

\section{Conclusion}

In summary, this quality improvement project had led to a significant improvement in healthcare services. It improved patient safety and quality of care, reduced cancellation rate at time of admission and improved utilisation of the theatre list. The changes that were made can also be easily applied in other hospitals nationwide to reduce cost on lost operating theatre time.

\section{Conflicts of interest}

None declared.

\section{Reference}

1 Gillies M. Counting the cost of cancelled surgery: a system wide approach is needed. Br J Anaesth 2018;121:694-7. https:// bjanaesthesia.org/article/S0007-0912(18)30643-3/pdf [Accessed 16 October 2019]. 